\title{
Biostatistical Analysis on the Consideration of Nutrition-Related Modules in the Higher Education Curriculum
}

\section{Zhao $\mathrm{B}^{1 *}$, Jiang $\mathrm{X}^{2}$, Cao J ${ }^{3}$, Li $\mathrm{Y}^{1}$ and Mingzhe $\mathrm{E}^{1}$}

${ }^{1}$ School of Science, Hubei University of Technology, China

${ }^{2}$ Hospital, Hubei University of Technology, China

${ }^{3}$ School of Information and Mathematics, Yangtze University, China

*Corresponding author: Bin Zhao, School of Science, Hubei University of Technology, Wuhan, Hubei, China, Tel/Fax: +86 1302851 7572; Email: zhaobin835@nwsuaf.edu.cn

\section{Research Article \\ Volume 4 Issue 1}

Received Date: February 06, 2020

Published Date: February 19, 2020

DOI: $10.23880 /$ jidtm-16000134

\section{Abstract}

Objective: To examine the impact of presenting healthy snack foods on the knowledge of the key recommendations for healthy eating patterns among university students.

Design: Cross sectional. The four-week intervention included the presentation of healthy snacks and informative leaflets to the participants. Each week featured a key recommendation of healthy eating (i.e. eat more dietary fibre) that was given in dietary guidelines. A nutrition knowledge questionnaire was administered pre and post-intervention. The questionnaire included some items from the General Nutrition Knowledge Questionnaire (GNKQ).

Setting: Participants were presented with the snacks and informative leaflets in their classrooms in their lecturers` presence. Test and re-test were taken under the same setting.

Participants: First year undergraduate students (n=378) studying a mandatory course at a remote campus.

Results: When the items belonging to the same theme (i.e. key recommendation) were grouped and analysed, no significant differences were observed in the distribution of responses (i.e. correct, incorrect and unsure) for "dietary fibre", "salt" and "sugar" after the intervention. However, the change in the distribution of the responses relevant to "less saturated fat and trans fat" was found to be statistically significant $(\mathrm{p}<0.05)$.

Conclusion: The intervention elicited positive response from the students and lecturers, who commented that the intervention made them more aware of healthy eating. There is a need for other nutrition education interventions (with longer durations) to improve eating behaviours of university students. We strongly recommend the consideration of nutrition-related modules in the higher education curriculum.

Keywords: Snack; Intervention; Nutrition Knowledge; University students

Abbreviations: GNKQ: General Nutrition Knowledge Questionnaire; BMI: Body Mass Index. 


\section{Introduction}

University students are vulnerable in their eating habits for a number of reasons such as obesity-promoting food environment, lack of nutrition knowledge, financial concerns and changes in the living arrangements [1-3]. Universities have a responsibility to provide a food environment that promotes healthy eating habits since students usually spend a substantial amount of time on campus $[4,5]$. It could be argued that there is an onus upon universities to implement nutritional educational programmes to support healthy food choices and encourage physical activity [6]. The term "snack foods" tends to connote energy-dense, nutrient-poor foods usually containing sugar, sodium, and/or saturated fat, for example cakes, cookies, chips and other salty snacks and sugar-sweetened beverages $[7,8]$. However, not all foods eaten as snacks are considered to be nutrient-poor [9]. Several of these foods such as fruits, nuts, yogurt, milk, low fat cheese and whole grain cereals can be a positive and nutritious element of eating patterns [10].

In addition to collective food knowledge, certain skills and behaviours are needed for healthy eating and these altogether make up the term "Food literacy" $[11,12]$. Due to the complex nature of food behaviour, nutritional knowledge on its own may not bring about the desired changes but it may still play a pivotal role in the adoption of healthier food habits [13]. Nutrition education interventions in improving university or college students' dietary intake are valuable tools and their contribution to healthy eating should not be underestimated [14]. Indeed, Bin Zhao [15] reported a number of intervention approaches that showed promise for improving college students' dietary habits.

This study examined the impact of presenting healthy snack foods on the knowledge of some key recommendations for healthy eating among university students. A preliminary survey, involving the same student cohort, identified a "lack of healthy eating choices for snacks" as the main reason for not increasing healthy food consumption whilst on campus [16]. Therefore snack foods have been chosen to convey the key recommendations. The intervention included the presentation of snack foods along with an informative leaflet that associated the healthy snack with a particular key recommendation. Nutrition knowledge associated with the recommendations was measured by a shortened version of a general nutrition knowledge questionnaire (GNKQ) [17].

\section{Methods}

\section{Participants}

Participants were first year undergraduate students studying a mandatory course from across seven different schools and scientific disciplines at a remote campus. None of the students have studied a food or nutrition- related module before or during the study. With $95 \%$ confidence level and 5\% confidence interval, out of 5403 undergraduate students, the sample size was found to be 359 . A total of 378 students participated in the intervention and completed the nutrition knowledge test (response rate 56.6\%). For the retest, the response rate was $26.2 \%$.

\section{Intervention}

The intervention, which took place in March-April 2017, included the preparation and presentation of healthy snacks and accompanying informative leaflets to the participants on a weekly basis for four weeks. Four key recommendations from the dietary guidelines that are listed below were assigned to each week of the intervention:

Week 1- Eat more dietary fibre

Week 2- Eat less sodium

Week 3- Eat less sugar

Week 4- Eat less saturated fats and trans-fats [18].

Nutrition knowledge test was administered before and after the intervention.

\section{Snack food preparation and nutritional analysis}

Snack foods were produced at a reasonable cost for students but offered free of charge during the intervention. The procedures for preparing the healthy snacks were as follows:

a) Reviewing current recipes of healthy snacks

b) Choosing healthy ingredients and modifying the recipes

c) Nutritional and cost analysis

d) Preparation of snacks

e) Storage and distribution of snacks

The healthy snacks produced were: Week 1- "hot-dry noodles" and "strawberry banana muffin"; week 2-"pumpkin and spinach muffin" and "crackers with seeds and yogurt dip"; week 3-"nut and dried fruit bar" and "strawberry banana smoothie"; week 4-"banana bread" and "bulgur (cracked wheat) served with yogurt". All ingredients were obtained by the purchasing department of the school. The snacks were prepared in the kitchens of the school by the researchers. They were individually packaged, labeled and stored at $4-8^{\circ} \mathrm{C}$ before being delivered to the classrooms. The classes, comprising of 8 to 35 students, were taught by different lecturers on different days and times. Lecturers were requested to encourage students to consume the snacks and read the leaflets at a convenient time (i.e. either during the lectures or at break times).

Informative Leaflets: Both sides of a coloured A4 size paper were used for each leaflet (Supplementary file 1). The leaflets were delivered to the classrooms together with the healthy snacks. Both were presented to the students whenever 
deemed suitable by lecturers.

Informative leaflets included:

a) Sources of the nutrient of concern (dietary fibre, salt, sugar etc.)

b) Effects of the nutrient on health

c) Healthy eating tips to increase or reduce the daily intake

d) Recommended daily amounts

e) The recipe and preparation method (these were included in an attempt to encourage students to prepare/cook their own food)

f) Nutrition labels, ingredient listing, production date and cost per portion (cost was included to demonstrate that it was possible to prepare healthy snacks at a reasonable price. Research has shown this was usually one of the most important factors determining the purchasing decision of snack foods in an educational setting [19]).

\section{Questionnaire}

The questionnaire items that are relevant to the themes of the intervention were taken from Bin Zhao [20]. There were 14 items relevant to dietary fibre, 13 items for salt, 12 items for sugar and 36 items for fat. There were 22 items that were not directly relevant to the themes but these were retained to measure some aspects of general nutrition knowledge before and after the intervention. The last part of the questionnaire included personal and anthropometric information (i.e. height and weight). The language of the questionnaire was English. The questionnaire was paperbased and administered in the presence of the lecturers. Participants completed the questionnaire anonymously.

\section{Ethical Approval}

Ethical approval was granted from the Ethics Committee of the University. Participation was voluntary and all participants filled in the 'Participation consent form'.

\section{Biostatistical Analysis}

Biostatistical analyses were conducted using the Statistical Package for Social Sciences, SPSS (IBMCorp. Released 2011. IBM SPSS Statistics for Windows, Version 20.0. Armonk, NY: IBM Corp.), with statistical significance set at $\mathrm{p}<0.05$. The raw data from each participant's responses were coded numerically. The responses were converted to 1 to 0 for correct and incorrect answers, respectively ('not sure' responses were also coded as incorrect). Descriptive statistics were used to analyse the demographic information and frequency tables. Parametric tests (t-test), nonparametric tests (Kruskal-Wallis test) and chi-square tests were used when necessary.

\section{Results}

\section{Participant Characteristics}

Among the participants who declared their gender (65.6\%), 36.2\% were male and $29.4 \%$ were female. The body mass index (BMI) of the participants was calculated by dividing the weight $(\mathrm{kg})$ by the square of the height (m2) and categorised as < 18.5 underweight, between 18.5 and 24.9 normal, between 25.0 and 29.9 overweight and $\geq$ 30 obese (18). It was found that $7.4 \%$ of the participants were underweight, 55.6\% were normal weight, 16.9\% were overweight and $4.5 \%$ were obese (data not shown) [21].

\section{Nutrition Knowledge before the Intervention}

Items answered correctly by most of the participants: The number and description of the most correctly answered items were: A8.1 Chicken has high protein content. This was the most correctly answered item (91.5\%). A27.1 Obesity is related to fat consumption (86.2\%). A10.1 Trans-fatty acid (TFA) content of margarine is high (85.4\%).

Items mostly answered incorrectly: A1.3 Most of the participants thought that experts suggested to eat more or the same amount of meat $(89.7 \%)$. However, dietary guidelines suggest a diet which reduces the amount of meat consumption while including a greater variety of other protein sources (legumes, seafood, dairy products and nuts) [18]. Moreover, the consumption of processed meat and meat products has been shown to cause cancer in humans [22] and this information has been disseminated widely by relevant media including the Ministry of Health publications.

A2 the majority of the participants (71.4\%) selected the option of eating less than the advised 5 portion of fruit and vegetables in a day, plus $18.3 \%$ of the participants were unsure of the correct answer. A previous study showed similar findings, where the adult participants knew the importance of increasing their intake of fruits and vegetables but were unaware of the number of daily portions [23].

A19 Most participants (61.1\%) answered the item about the energy contents of the foods incorrectly. $30.2 \%$ of participants thought that simit (type of bagel covered with sesame seeds) had the lowest energy content (when compared to baked apple, strawberry yogurt and vanilla ice cream). This was similar to the findings of another study where most participants answered the item on the energy content of the macronutrients incorrectly [23].

Items participants were mostly unsure of: A24.5 $53.2 \%$ of the participants was unsure of the relationship between cirrhosis and the intake of fruit and vegetables. A3 $48.7 \%$ were unsure which type of fat (monounsaturated, 
polyunsaturated and saturated) experts say is the most important for people to reduce intake. This item measured 'factual/declarative knowledge' and it contained scientific terms that the participants were mostly unfamiliar with. A27.4 45.0\% was unsure whether there was a relationship between constipation and fat consumption.

\section{Nutrition Knowledge after the Intervention}

The following items, whose correct answers were listed below, had a significant change in the distribution of responses (correct, incorrect and unsure) after the intervention (Table 1).

\begin{tabular}{|c|c|c|c|c|c|c|c|}
\hline \multirow{3}{*}{ Themes } & \multirow{3}{*}{ Item number } & \multicolumn{6}{|c|}{ Scores (\%) } \\
\hline & & \multicolumn{3}{|c|}{ Before } & \multicolumn{3}{|c|}{ After } \\
\hline & & Correct & Incorrect & Unsure & Correct & Incorrect & Unsure \\
\hline \multirow{4}{*}{ Dietary fibre } & $\mathrm{A} 24.1$ & $126(33.3)$ & $33(8.7)$ & $196(51.9)$ & $80(45.7)$ & $22(12.6)$ & $58(33.1)$ \\
\hline & A24.3 & $170(45.0)$ & $66(17.5)$ & $120(31.7)$ & $94(53.7)$ & $28(16.0)$ & $38(21.7)$ \\
\hline & $\mathrm{A} 24.4$ & $65(17.2)$ & $148(39.2)$ & $140(37.0)$ & $29(16.6)$ & $88(50.3)$ & $42(24.0)$ \\
\hline & $\mathrm{A} 24.5$ & $80(21.2)$ & $70(18.5)$ & $201(53.2)$ & $43(24.6)$ & $46(26.3)$ & $71(40.6)$ \\
\hline Salt & A7.3 & $132(34.9)$ & $65(17.2)$ & $166(43.9)$ & 87 (49.7) & $26(14.9)$ & $55(31.4)$ \\
\hline \multirow{2}{*}{ Sugar } & A5.1 & $212(56.1)$ & $157(41.6)$ & $102(27.0)$ & $104(59.4)$ & $34(19.4)$ & $29(16.6)$ \\
\hline & A13 & $93(24.6)$ & $240(63.5)$ & $34(9.0)$ & $51(29.1)$ & $90(51.4)$ & $28(16.0)$ \\
\hline \multirow{5}{*}{ Saturated fat and trans- fat } & A3 & $106(28.0)$ & $70(18.5)$ & $184(48.7)$ & $67(38.3)$ & $26(14.9)$ & $80(45.7)$ \\
\hline & A6.1 & $161(42.6)$ & $186(49.2)$ & $23(6.1)$ & $93(53.1)$ & $65(37.1)$ & $14(8.0)$ \\
\hline & A11.4 & $186(49.2)$ & $98(25.9)$ & $74(19.6)$ & $108(61.7)$ & $29(16.6)$ & 32 (18.3) \\
\hline & A27.4 & $92(24.3)$ & $91(24.1)$ & $170(45.0)$ & $25(14.3)$ & $55(31.4)$ & $69(39.4)$ \\
\hline & A28.4 & $247(65.3)$ & $64(16.9)$ & 48 (12.7) & $117(66.9)$ & $18(10.3)$ & $15(8.6)$ \\
\hline \multirow{4}{*}{ General items } & A1.1 & $285(75.4)$ & $89(23.6)$ & $2(0.5)$ & $149(85.1)$ & $23(13.1)$ & $2(1.1)$ \\
\hline & A1.3 & $22(5.8)$ & 339 (89.7) & $13(3.4)$ & $27(15.4)$ & $143(81.7)$ & $3(1.7)$ \\
\hline & $\mathrm{A} 2$ & $27(7.1)$ & $270(71.4)$ & $69(18.3)$ & $25(14.3)$ & $123(70.3)$ & $23(13.1)$ \\
\hline & A15 & $73(19.3)$ & 139 (36.8) & $155(41.0)$ & $47(26.9)$ & $57(32.6)$ & $65(37.1)$ \\
\hline
\end{tabular}

Table 1: Items whose score distribution changed significantly $(\mathrm{p}<0.05)$ after the intervention.

\section{Dietary Fibre (4 items)}

Constipation (24.1) and common cold (24.3) are related to low intake of fruit and vegetables; whereas dental caries (24.4) and cirrhosis (24.5) are not.

\section{Salt (1 item)}

Kippers are high in salt (A7.3).

\section{Sugar (2 items)}

Pomegranate sauce (processed, not fresh) is high in sugar (A5.1). Brown sugar is not a healthy alternative to white sugar (A13).

\section{Saturated fat and trans-fat (5 items)}

Saturated fat is the most important for people to reduce intake (as recommended by experts) (A3).

Pasta without sauce is low in fat (A6.1).
Cheddar cheese is high in saturated fat (A11.4).

Constipation is not related to fat consumption (A27.4).

Eating less saturated fat can prevent heart disease (A28.4).

\section{General items (3 items)}

Health experts recommend that people should be eating more vegetables (A1.1).

Experts are advising people to eat 5 servings of fruits and vegetables a day (A2).

There is not more calcium in a glass of whole milk than a glass of skimmed milk (A15).

When the items belonging to the same theme were grouped and analysed, no significant differences were observed in the distribution of responses (i.e. correct, incorrect and unsure) for "dietary fibre", "salt", "sugar" and 
"general items" after the intervention (Table 2). However, the change in the distribution of the responses relevant to "less saturated fat and trans-fat" was found to be biostatistically significant $(\mathrm{p}<0.05) .21$ items $(58.3 \%)$ within this category had an increase in the correctresponses after the intervention.

\begin{tabular}{|c|c|c|c|}
\hline \multirow{2}{*}{ Intervention theme (major item no) } & \multicolumn{3}{|c|}{ Total score (\%) } \\
\hline & Response & Before & After \\
\hline \multirow{2}{*}{ Dietary fibre (A9, A17, A24) } & Correct & $923(48.1)$ & $998(52.0)$ \\
\hline & Incorrect & $1200(52.5)$ & $1087(47.5)$ \\
\hline \multirow{2}{*}{ Salt (A4, A7, A20, A26) } & Correct & $1003(50.2)$ & $996(49.8)$ \\
\hline & Incorrect & $983(51.3)$ & $933(48.7)$ \\
\hline \multirow{2}{*}{ Sugar (A5, A13, A18, A25) } & Correct & $1010(51.2)$ & $963(48.8)$ \\
\hline & Incorrect & $840(50.2)$ & $834(49.8)$ \\
\hline \multirow{2}{*}{ Fat *(A3, A6, A10, A11, A16, A17, A22, A27, A28.4) } & Correct & $2850(49.1)$ & $2960(51.0)$ \\
\hline & Incorrect & $2617(52.9)$ & $2330(47.1)$ \\
\hline \multirow{2}{*}{ General items (A1, A2,A8, A12, A14, A15, A19, A21, A23) } & Correct & $1707(48.9)$ & $1781(51.1)$ \\
\hline & Incorrect & $1659(51.8)$ & $1542(48.2)$ \\
\hline \multicolumn{4}{|c|}{$*$ denotes biostatistical significance $(\mathrm{p}<0.05)$} \\
\hline
\end{tabular}

Table 2: Total scores by theme - before and after intervention.

The question items relevant to "less saturated fat and transfat" included:

a) Experts` recommendation of type of fat to cut down

b) Identification of foods that are high and low in fat

c) Identification of foods that contain trans-fatty acids

d) Comparison of the saturated fatty acid content of fats and oils,

e) The best choice for a low fat-high fibre snack,

f) Comparison of the percent energy provided by butter versus sunflower oil

g) Diseases related to fat consumption and

h) Eating less saturated fat preventing heart disease.

Some items in Table 1 were incorrectly answered by most of the participants even after the intervention. These items were A1.3 (81.7\%), A2 (70.3\%) and A13 (51.4\%). Item A1.3 (experts recommend people to eat less meat) was not referred to in any of the leaflets. Item A2 (eating 5 servings of fruits and vegetables) was mentioned briefly at the end of each leaflet as a reminder. Due to the repetition of this information, a greater percentage of correct answers were anticipated than obtained. Item A13 (brown sugar is a healthy alternative to white sugar) was mentioned once in the "Eat less sugar" leaflet. This specific information possibly did not catch the attention of the participants.

\section{Discussion}

The intervention resulted in an improvement only in the knowledge scores of the theme "less saturated fat and transfat" $(p<0.05)$. A possible reason for incorrect/unsure answers post-intervention for the other themes (dietary fibre, sugar and salt) could be due to the participants not converting the information available on the leaflet into declarative/factual knowledge (i.e. knowledge of what is) [13]. As previously discussed, gaining factual information is not always sufficient to actualise healthy eating habits. Consumers may need to transform this knowledge into procedural knowledge (i.e. knowledge of how to) to perform food behaviours such as knowing how to choose the healthier of two snacks or how to compose a balanced menu [24].

Regular consumption of fruit and vegetables was reminded in each leaflet but the intervention only caused $7.2 \%$ increase in the correct answers (regarding the serving size). Although it may not be appropriate to use one item to assess the efficiency of the intervention, this made us question whether communicating the information in the leaflets by online tools (such as e-mails or social media) would have been more efficient and less time- constraining. Digital nutrition interventions such as internet programming and mobile phone applications have started to be promising future research directions as they are relatively costeffective and efficient [15]. A recent study portrays an interesting example of how digital technology can be used to collect nutritional information. Using a compact wristworn electronic food diary, snacking data, location and social context are recorded in real time [25].

Only $26.0 \%$ of students were reported to have received nutrition education prior to graduation from high school 
in China [26]. Therefore, engagement with educational resources (academic courses, peer learning, extracurricular activities, meeting with nutritional professionals and getting free food from events) in higher education settings may be effective in improving food literacy. Academic courses in higher education settings have been reported to be effective in changing food behaviour [15]. Malan $\mathrm{H}$, et al. [27] stated that students found the information provided by professors, dietitians and the university itself useful and credible. We therefore suggest the consideration of nutrition-related modules to be accommodated within the higher education curriculum - even when offered initially as optional modules.

The reason why nutrition labels were produced for the snack foods was to emphasise the importance of reading labels and to encourage their use. The preliminary results showed that the buying decision of $48.7 \%$ of participants (for snack foods sold on the campus) was affected by the presence of nutritional labels on packaged foods $(n=320)$ [16]. This value was less than the value reported $(72.4 \%)$ in a study, which recruited Chinese consumers aged 12 to $54(n=1536)$ [28]. Fidelity in intervention ensures that all intervention activities are executed as planned in the methods [29]. As none of the researchers were present at the time when the snacks and leaflets were presented, we were not able to verify that all students were encouraged or given adequate time to read the leaflets and taste the snacks. It is possible that lack of intervention fidelity could have compromised the findings.

Duration of the nutrition education interventions is one of the factors affecting their efficiency [29]. It is suggested that interventions lasting for greater than 5 months reported higher levels of success [30]. In this study, the intervention only encompassed 4 weeks, which was less than the recommended time period. The first administration of the questionnaire had a response rate of $56.6 \%$, however for the re-test, this rate declined to $26.6 \%$. Spring break, which coincided with the 4 th week of the intervention, resulted in a considerable decrease in the attendance level of students. A control group was not used in the questionnaire. Only one university was examined and therefore the results should be used with caution. Finally, as with any type of survey, participants who intentionally or unintentionally distorted their responses may represent a source of bias.

\section{Conclusion}

The intervention elicited positive response from the students and lecturers. The participants commented that the intervention made them more aware of healthy eating. The intervention was useful in creating an agenda for decision-makers of the university to adopt organisational changes including those that increase the availability and affordability of foods and snacks that align more closely with the key recommendations. There is a need for other nutrition education interventions (with longer durations) to improve eating behaviour of Chinese university students. We strongly recommend the consideration of nutrition-related modules in the higher education curriculum.

\section{Conflict of Interest}

We have no conflict of interests to disclose and the manuscript has been read and approved by all named authors.

\section{Acknowledgment}

This work was supported by the Philosophical and Social Sciences Research Project of Hubei Education Department (19Y049), and the Staring Research Foundation for the Ph.D. of Hubei University of Technology (BSQD2019054), Hubei Province, China.

\section{References}

1. Zhao B (2017) Research on Biomathematics Thought. $1^{\text {st }}$ (Edn.), Science Publishing House, Beijing, China, pp: 3336.

2. Zhao B (2017) Research on Biomathematics Thought. $1^{\text {st }}$ (Edn.), Science Publishing House, Beijing, China, pp: 4248.

3. Roy R, Kelly B, Rangan A, Allman-Farinelli M (2015) Food environment interventions to improve the dietary behavior of young adults in tertiary education settings: A systematic literature review. J Acad Nutr Diet 115(10): 1647-1681.

4. Tam R, Yassa B, Parker H, O Connor H, Allman-Farinelli M (2017) University students' on-campus food purchasing behaviors, preferences, and opinions on food availability. Nutrition 37: 7-13.

5. Roy R, Soo D, Conroy D, Wall CR, Swinburn B (2019) Exploring university food environment and on-campus food purchasing behaviors, preferences, and opinions. J Nutr Educ Behav 51(7): 865-875.

6. Freedman MR (2010) Development, evaluation, and validation of environmental assessment tools to evaluate the college nutrition environment. J Am Coll Health 58(6): 565-568.

7. Zhao B (2017) Research on Biomathematics Thought. $1^{\text {st }}$ (Edn.), Science Publishing House, Beijing, China, pp: 5357. 
8. Bellisle F (2014) Meals and snacking, diet quality and energy balance. Physiology \& Behavior 134: 38-43.

9. Hess JM, Slavin JL (2018) The benefits of defining "snacks." Physiol Behav 193: 284-287.

10. Zhao B (2017) Research on Biomathematics Thought. ${ }^{\text {st }}$ (Edn.), Science Publishing House, Beijing, China, pp: 6263.

11. Vidgen HA, Gallegos D (2014) Defining food literacy and its components. Appetite 76: 50-59.

12. Truman E, Lane D, Eliott C (2017) Defining food literacy: A scoping review. Appetite 116: 365-371.

13. Worsley A (2002) Nutrition knowledge and food consumption: can nutrition knowledge change food behavior? Asia Pac J Clin Nutr 11(3): 579-585.

14. Deliens $T$, Van Crombruggen $R$, Verbruggen $S$, De Bourdeaudhuij I, Deforche B, et al. (2016) Dietary interventions among university students: A systematic review. Appetite 105: 14-26.

15. Zhao B (2017) Research on Biomathematics Thought. $1^{\text {st }}$ (Edn.), Science Publishing House, Beijing, China, pp: 6475.

16. Zhao B (2017) Research on Biomathematics Thought. $1^{\text {st }}$ (Edn.), Science Publishing House, Beijing, China, pp: 6667.

17. Zhao B (2017) Research on Biomathematics Thought. $1^{\text {st }}$ (Edn.), Science Publishing House, Beijing, China, pp: 6870.

18. Zhao B (2017) Research on Biomathematics Thought. $1^{\text {st }}$ (Edn.), Science Publishing House, Beijing, China, pp: 7174.

19. Buscher LA, Martin KA, Crocker S (2001) Point-ofpurchase messages framed in terms of cost, convenience, taste, and energy improve healthful snack selection in a college foodservice setting. J Am Diet Assoc 101(8): 909913.
20. Zhao B (2017) Research on Biomathematics Thought, $1^{\text {st }}$ (Edn.), Science Publishing House, Beijing, China, pp: 7576.

21. Zhao B (2017) Research on Biomathematics Thought. $1^{\text {st }}$ (Edn.), Science Publishing House, Beijing, China, pp: 7879.

22. Zhao B (2017) Research on Biomathematics Thought. $1^{\text {st }}$ (Edn.), Science Publishing House, Beijing, China, pp: 8183.

23. Zhao B (2017) Research on Biomathematics Thought. $1^{\text {st }}$ (Edn.), Science Publishing House, Beijing, China, pp: 8587.

24. Dickson-Spillman M, Siegrist M (2011) Consumers knowledge of healthy diets and its correlation with dietary behaviour. J Hum Nutr Diet 24(1): 54-60.

25. Allan J, McMinn D, Powell D (2019) Tracking snacking in real time: Time to look at individualised patterns of behaviour. Nutr Health 25(3): 179-184.

26. Zhao B (2017) Research on Biomathematics Thought. $1^{\text {st }}$ (Edn.), Science Publishing House, Beijing, China, pp: 8889.

27. Malan H, Watson TD, Slusser W, Glik D, Rowat AC, et al. (2020) Challenges, opportunities, and motivators for developing and applying food literacy in a university setting: A qualitative study. J Acad Nutr Diet 120(1): 3344.

28. Zhao B (2017) Research on Biomathematics Thought. $1^{\text {st }}$ (Edn.), Science Publishing House, Beijing, China, pp: 9294.

29. Murimi MW, Kanyi M, Mupfudze T, Amin MR, Mbogori T, et al. (2017) Factors influencing efficacy of nutrition education interventions: A systematic review. J Nutr Educ Behav 49(2): 142-165.

30. Zhao B (2017) Research on Biomathematics Thought. $1^{\text {st }}$ (Edn.), Science Publishing House, Beijing, China, pp: 9598. 